\title{
Metal-free visible-light-enabled vicinal trifluoromethyl dithiolation of unactivated alkenes
}

\author{
Xiaojuan $\mathrm{Li}^{1}$, Qiang Zhang ${ }^{1}$, Weigang Zhang ${ }^{*}$, Jinzhu Ma ${ }^{* 2}$, Yi Wang ${ }^{* 1}$ and Yi Pan ${ }^{1}$
}

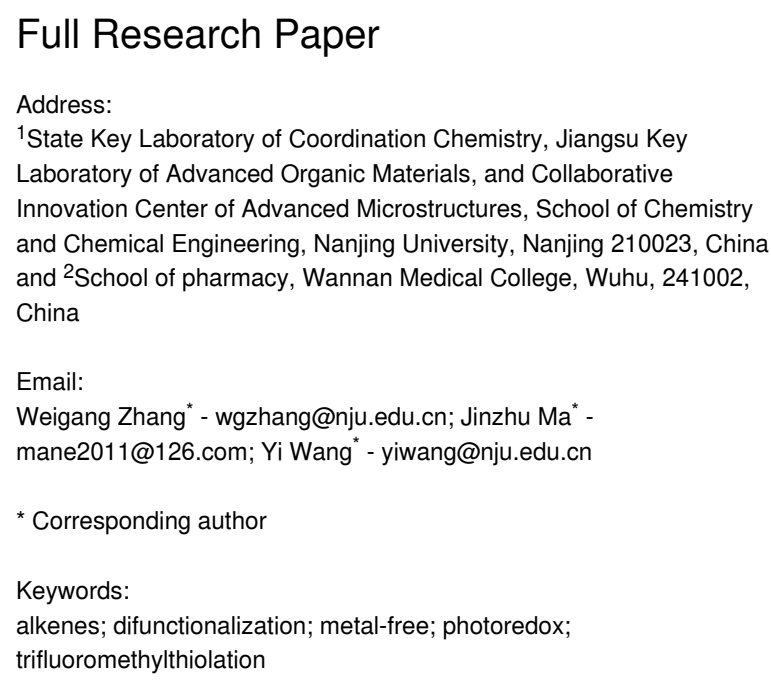

trifluoromethylthiolation

Beilstein J. Org. Chem. 2021, 17, 551-557.

https://doi.org/10.3762/bjoc.17.49

Received: 13 January 2021

Accepted: 12 February 2021

Published: 24 February 2021

This article is part of the thematic issue "Organo-fluorine chemistry V".

Guest Editor: D. O'Hagan

(c) 2021 Li et al.; licensee Beilstein-Institut. License and terms: see end of document.

\begin{abstract}
The difunctionalization of alkenes involving a trifluoromethylthio group $\left(\mathrm{SCF}_{3}\right)$ for the conversion of versatile and readily available olefins into structurally more complex molecules has been successfully studied. However, the disproportionate dithiolation of alkenes is unknown. Herein, a transition-metal-free protocol is presented for the vicinal trifluoromethylthio-thiolation of unactivated alkenes via a radical process under mild conditions with a broad substrate scope and excellent tolerance.
\end{abstract}

\section{Introduction}

The incorporation of fluorine atoms into drug molecules will significantly enhance the physical, chemical, and biological properties of the pharmaceuticals [1-6]. Modifying drug candidates by introducing fluorine-containing (such as $-\mathrm{CF}_{3},-\mathrm{CF}_{2} \mathrm{H}$, $\left.-\mathrm{C}_{2} \mathrm{~F}_{5},-\mathrm{SCF}_{3},-\mathrm{OCF}_{3}\right)$ moieties has become a substantial strategy for medicinal research $[2,7,8]$. Among the fluorinated functionalities, the trifluoromethylthio group $\left(\mathrm{SCF}_{3}\right)$ has strong electron-withdrawing properties and a higher lipophilicity $\left(\pi_{\mathrm{R}}=1.44\right)$, compared with $\mathrm{CF}_{3}\left(\pi_{\mathrm{R}}=0.88\right)$ and $\mathrm{SCH}_{3}$ $\left(\pi_{R}=0.61\right)$, which could improve the pharmaceuticals' ability to cross lipid membranes $[9,10]$. Along these lines, the introduc- tion of the $\mathrm{SCF}_{3}$ group into small molecules has attracted great attention in organofluorine methodology [11-17].

The vicinal difunctionalization of olefins to introduce two functional groups across a double bond has appeared as a powerful transformation to rapidly increase molecular complexity in synthetic chemistry with improved efficiency [18-22]. Various transition-metal-mediated approaches for the trifluoromethylthio $\left(\mathrm{SCF}_{3}\right)$ difunctionalization of alkenes, such as cyanation [23], etherification [24-27], amination [28-30], chlorination [31,32], hydrogenation [33], trifluoromethylation [34], phos- 
phonization [35], arylation [36-38], trifluoromethylthiolation [39], fluorination [40], and selenylation [41] have been reported (Scheme 1a). However, the visible-light-induced trifluoromethylthio difunctionalization of alkenes remained underdeveloped. For instance, Magnier and co-workers have documented a practical intramolecular carbotrifluoromethylthiolation of acrylamides under irradiation of visible light [38]. In 2017, the photoredox-catalyzed intermolecular trifluoromethylthio-trifluoromethylation and thiosulfonylation reaction of unactivated alkenes have been respectively developed by Liu [34] and Xu [42]. Recently, Qing [43] and co-workers reported an efficient anti-Markovnikov hydrotrifluoromethylthiolation of alkenes utilizing trifluoromethanesulfonic anhydride $\left(\mathrm{Tf}_{2} \mathrm{O}\right)$ as a radical trifluoromethylthiolating reagent through a deoxygenative reduction and a photoredox radical pathway (Scheme 1b). The $\mathrm{C}-\mathrm{S}$ bond $[44,45]$ is an important structural motif that is widely present in natural products, drug molecules, biologically active molecules, and functional materials. However, the highly selective incorporation of two different sulfur-bearing moieties across double bonds remains challenging [42]. Herein, we describe a visible-light-enabled cascade radical difunctionalization of unactivated alkenes for the construction of partially trifluoromethylated dithioethers with a broad substrate scope and a good chemical tolerance (Scheme 1c).

\section{Results and Discussion}

We evaluated the reaction conditions for this trifluoromethylthio-thiolation and found out that under irradiation of blue
LEDs, allyl boronate 1a $(0.1 \mathrm{mmol})$, disulfide $2 \mathbf{a}$ (1.0 equiv) and $\mathrm{N}$-(trifluoromethylthio)phthalimide (Phth- $\mathrm{SCF}_{3}, \mathbf{3}$, 1.5 equiv), the desired trifluoromethylthiolated product $\mathbf{4 a}$ was obtained in 71\% yield with 1,2,3,5-tetrakis(carbazol-9-yl)-4,6dicyanobenzene (4CzIPN, $2 \mathrm{~mol} \%$ ) as the photocatalyst and $\mathrm{KH}_{2} \mathrm{PO}_{4}(10 \mathrm{~mol} \%)$ as the base (Table 1, entry 1$)$. The yield of $4 \mathbf{a}$ was not increased when 2 equiv of $\mathrm{K}_{2} \mathrm{HPO}_{4}$ were used (Table 1, entry 2) and no difunctionalized product was observed with DMA as the solvent (Table 1, entry 4). The employment of $\mathrm{KH}_{2} \mathrm{PO}_{4}$ as base and $\left[\operatorname{Ir}\left(\mathrm{dF}\left(\mathrm{CF}_{3}\right) \mathrm{ppy}\right)_{2}\right.$ (dtbby) $] \mathrm{PF}_{6}$ as the photocatalyst furnished the product in very low yields (Table 1, entries 3 and 5). The control experiments indicated that $4 \mathrm{CzIPN}, \mathrm{K}_{2} \mathrm{HPO}_{4}$ or visiblelight were indispensable for the reaction to proceed (Table 1, entries 6-8).

With the optimized reaction conditions determined, we next examined the substrate scope of the disulfides (Scheme 2). Using borate-substituted olefins, the intermolecular trifluoromethylthio-thiolation induced by the sequential radical difunctionalization proceeded smoothly in a chemoselective fashion. Both, with electron-withdrawing and electron-donating groups substituted aryldisulfides were tolerated to access products $\mathbf{4 a - g}$. The homoallylic borate (1b) was also converted into the corresponding product $\mathbf{4 h}$ in a moderate yield.

In order to further examine the generality of the reaction, we have extended this protocol to a range of unactivated alkenes.

previous work:

(a) conventional methods for trifluoromethylthiolation of alkenes

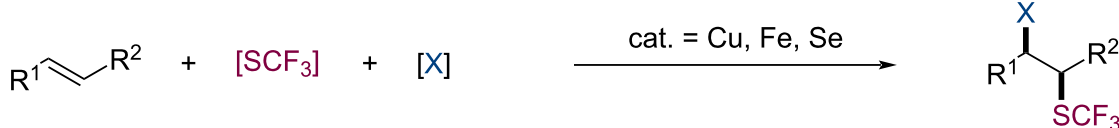

$$
\begin{aligned}
& X=-C N,-O R,-N_{2},-C l,-H,-S_{3},-P O(O E t)_{2},-A r,-F,-S e P h
\end{aligned}
$$

(b) visible light-mediated trifluoromethylthiolation of alkenes

$$
\begin{aligned}
& \mathrm{R}^{1} \mathrm{R}^{2}+\left[\mathrm{SCF}_{3}\right]+[\mathrm{Y}] \longrightarrow \mathrm{PC}, h v+\mathrm{R}^{1} \overbrace{\mathrm{SCF}_{3}}^{\mathrm{R}^{2}} \\
& \mathrm{Y}=-\mathrm{Ar},-\mathrm{H},-\mathrm{CF}_{3},-\mathrm{SO}_{2} \mathrm{Ph}
\end{aligned}
$$

(c) this work: photoredox-catalyzed trifluoromethylthio-thiolation of alkenes

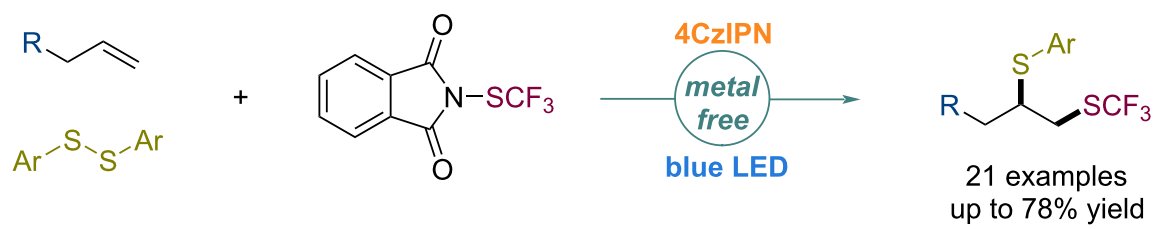


Table 1: Optimization of the reaction conditions. ${ }^{a}$

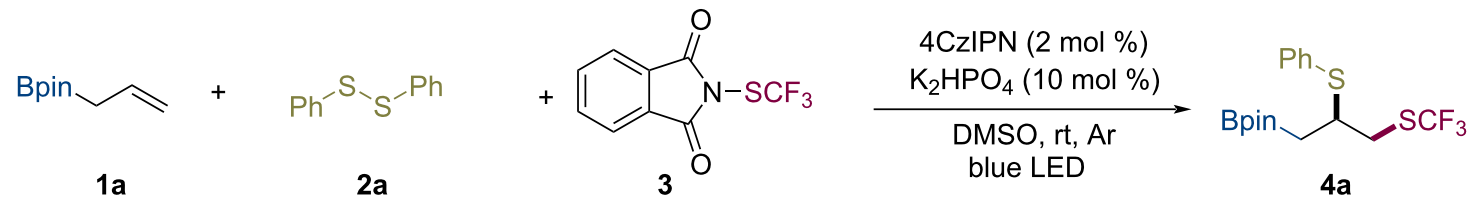

\begin{tabular}{lll}
\hline entry & deviation from the reaction conditions & yield $(\%)$ \\
\hline 1 & none & 71 \\
2 & 2.0 equiv $\mathrm{K}_{2} \mathrm{HPO}_{4}$ & 70 \\
3 & $\mathrm{KH}_{2} \mathrm{PO}_{4}$ instead of $\mathrm{K}_{2} \mathrm{HPO}_{4}$ & 34 \\
4 & $\mathrm{DMA}$ instead of $\mathrm{DMSO}^{\mathrm{b}}$ & trace \\
5 & {$\left[\mathrm{Ir}\left(\mathrm{dF}\left(\mathrm{CF}_{3}\right) \mathrm{ppy}\right)_{2}(\mathrm{dtbby})\right] \mathrm{PF}_{6}$ instead of $4 \mathrm{CzIPN}$} & 43 \\
6 & no $\mathrm{K}_{2} \mathrm{HPO}_{4}$ & 0 \\
7 & without $4 \mathrm{CzIPN}$ & 0 \\
8 & in darkness & 0 \\
\hline
\end{tabular}

aReaction conditions: 1a (0.1 mmol), 2a (1.0 equiv), 3 (1.5 equiv), 4CzIPN (2 mol \%), $\mathrm{K}_{2} \mathrm{HPO}_{4}(10 \mathrm{~mol} \%)$, rt, Ar, $24 \mathrm{~h}$. ${ }^{\mathrm{b}} \mathrm{Crude}$ yields were determined by ${ }^{19} \mathrm{~F}$ NMR using trifluoromethoxybenzene as an internal standard.

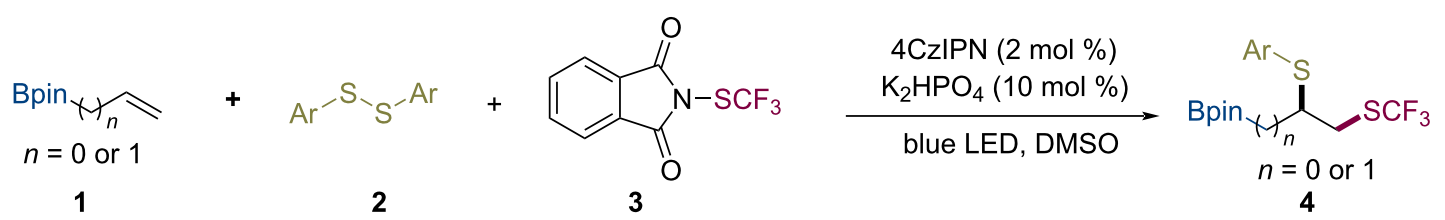

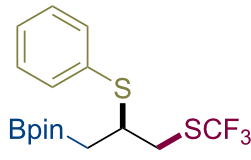

4a, $70 \%$<smiles>FC(F)(F)[Se]CC(C[Se]C(F)(F)F)Sc1cc(Cl)cc(Cl)c1</smiles>

$4 e, 65 \%$<smiles>CC(C)(C)c1ccc(SC(CBr)CSC(F)(F)F)cc1</smiles>

4b, $60 \%$<smiles>Fc1ccccc1SC(CBr)CSC(F)(F)F</smiles>

$4 f, 55 \%$<smiles>Cc1ccc(SC(C[AsH2]C(F)(F)F)Cc2ccccc2)cc1</smiles>

4c, $68 \%$<smiles>FC(F)(F)SCC(CBr)Sc1ccc(Cl)cc1</smiles>

4g, $68 \%$<smiles>FC(F)(F)CC(CBr)CSc1ccc(Br)cc1</smiles>

4d, $58 \%$

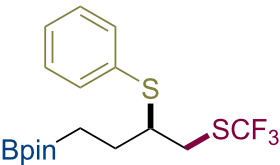

4h, $40 \%$

Scheme 2: Substrate scope of disulfides.

Terminal alkenes containing ester (5a-d) and oxygenated alkyl $(\mathbf{5 e}-\mathbf{g})$ functionalities were tolerated under this framework (Scheme 3). The tested 1-phenyl-3-butene substrates transformed into the desired products $\mathbf{5} \mathbf{h}$ and $\mathbf{5 i}$ in good yields. Also olefins containing amido $(\mathbf{5 j})$ and sulfonate $(\mathbf{5 k})$ functionalities furnished the corresponding $\mathrm{SCF}_{3}$ adducts. In addition, boldenone- and D-glucose-derived terminal alkenes were compatible with the conditions affording the corresponding products $\mathbf{5} \mathbf{l}$ and $\mathbf{5 m}$ in moderate to good yields.

To gain insight into the reaction mechanism, control experiments were conducted under the standard conditions (Scheme 4). The radical-trapping agent 2,2,6,6-tetramethylpiperidin-1-oxyl (TEMPO, 3.0 equiv) completely prevented the 

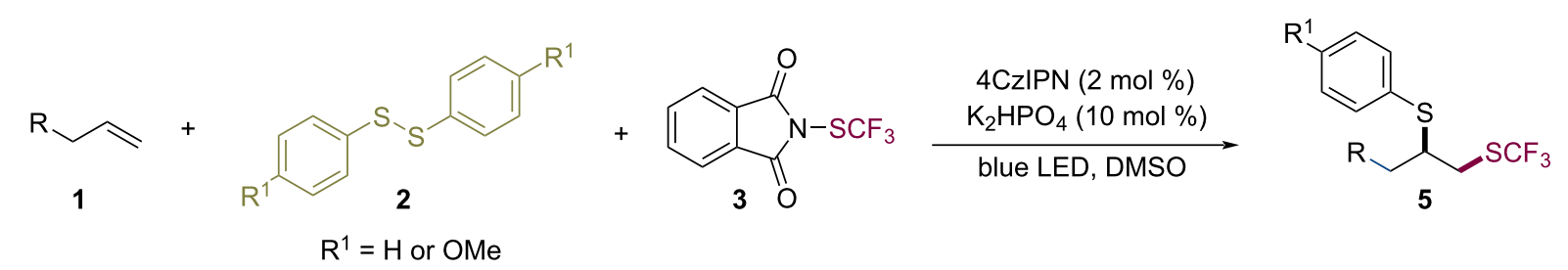<smiles>O=C(OCCCC(CC(F)(F)F)Sc1ccccc1)c1ccc(Cl)cc1</smiles>

5a, $78 \%$<smiles>O=C(OCCCCC(C[Si](F)(F)F)c1ccccc1)c1ccc2ccccc2c1</smiles><smiles>Cc1ccc(C(=O)OCCCCC(CC(F)(F)F)Sc2ccccc2)cc1</smiles>

5b, $66 \%$<smiles>COc1cc(C(=O)OCCCC(CC(F)(F)F)Sc2ccccc2)cc([N+](=O)[O-])c1</smiles>

5c, $72 \%$ 5d, $67 \%$<smiles>COc1cccc(OCCC(COC(F)(F)F)SPc2ccccc2)c1</smiles>

5g, $67 \%$<smiles>F[C-](F)CC(CCc1ccccc1)Sc1ccccc1</smiles>

5h, $78 \%$<smiles>Brc1cccc(OCCCC(CSc2ccccc2)Sc2ccccc2)c1</smiles>

5e, $61 \%$<smiles>O=c1c(OCCCC(C[As](F)(F)F)Sc2ccccc2)c(-c2ccccc2)oc2ccccc12</smiles>

5f, $65 \%$<smiles>COc1ccc(SC(C[SeH2])CCc2ccccc2)cc1</smiles>

5i, $71 \%$<smiles>O=C1c2ccccc2C(=O)N1CCCC(F)(F)C[Ge]F</smiles>

5j, $68 \%$<smiles>Cc1ccc(S(=O)(=O)OCCCCC(CSCC(F)(F)F)Sc2ccccc2)cc1</smiles>

(a)<smiles>C=CCCc1ccccc1</smiles>

6 $\underset{\text { TEMPO (3.0 equiv) }}{\stackrel{\text { standard conditions }}{\longrightarrow}}$ 3 $2 a$<smiles>C=C(c1ccccc1)c1ccccc1</smiles><smiles>F[Si]([Si])C=C(c1ccccc1)c1ccccc1</smiles>

7, $12 \%{ }^{19} \mathrm{~F}$ NMR yield and detected by GCMS<smiles>FC(F)(F)CCC(Cc1ccccc1)Sc1ccccc1</smiles>

5h, $0 \%$ (b) $6+2 a+3 \frac{\mathrm{Ph}_{\mathrm{Ph}}}{\text { standard conditions }}$

Scheme 4: Control experiments. 
reaction. When diphenylethylene (3.0 equiv) was added to the reaction mixture, the desired product $\mathbf{5 h}$ was not obtained, and the vinyltrifluoromethylsulfide 7 was afforded in $12 \%$ yield $[46,47]$.

Based on the above results and previous literature reports $[33,38,48]$, a plausible mechanism for the trifluoromethylthio-thiolation is proposed (Scheme 5). In solution, two resonance structures can be formulated for Phth- $\mathrm{SCF}_{3}, 3$ $\left(E_{1 / 2}{ }^{\text {red }}=-0.45 \mathrm{~V}\right.$ vs SCE) [49] and the intermediate $\mathbf{I}$ [50] The complexation of the intermediate I with $\mathrm{K}_{2} \mathrm{HPO}_{4}$ provides the intermediate II. Then, $4 \mathrm{CzIPN}^{*}\left(4 \mathrm{CzIPN}^{+} / 4 \mathrm{CzIPN} *\right.$ : $E_{1 / 2}$ red $=-1.04 \mathrm{~V}$ vs SCE) [51] reduces the intermediate II to generate a phthalimide anion $\left(\mathrm{Phth}^{-}[50]\right)$ and a trifluoromethylthio radical under irradiation. The $\mathrm{SCF}_{3}$ radical readily reacts with the unactivated alkenes to give the intermediate $\mathbf{B}$. The subsequent addition of $\mathbf{B}$ to disulfide $\mathbf{2}$ affords the difunctionalized products $\mathbf{4}$ or $\mathbf{5}$ and the thiophenyl radical $\mathbf{C}$ [52]. Finally, the oxidation of radical $\mathbf{C}$ by $4 \mathrm{CzIPN}^{+}$closes the catalytic cycle [53].

\section{Conclusion}

In summary, we described a visible-light-induced cascade radical difunctionalization of unactivated alkenes to provide partially trifluoromethylated dithioethers. The approach features practical conditions, good functional group tolerance, and a broad substrate scope allowing the incorporation of two distinctive sulfur-containing motifs into terminal olefins.

\section{Supporting Information}

\section{Supporting Information File 1}

Full experimental details, compound characterization, and copies of NMR spectra.

[https://www.beilstein-journals.org/bjoc/content/ supplementary/1860-5397-17-49-S1.pdf]

\section{Funding}

We gratefully acknowledge the financial support from the National Natural Science Foundation of China (Nos. 21772085, 21971107, 22071101) and the Fundamental Research Funds for the Central Universities (Nos. 020514380220, 020514380131, 020514913412, 020514913214). We also thank the Collaborative Innovation Center of Advanced Microstructures and the Jiangsu Provincial Key Laboratory of Photonic and Electronic Materials at Nanjing University for support.

\section{ORCID ${ }^{\circledR}$ iDs}

Jinzhu Ma - https://orcid.org/0000-0002-0581-3137

Yi Wang - https://orcid.org/0000-0002-8700-7621

\section{Preprint}

A non-peer-reviewed version of this article has been previously published as a preprint: https://doi.org/10.3762/bxiv.2021.1.v1

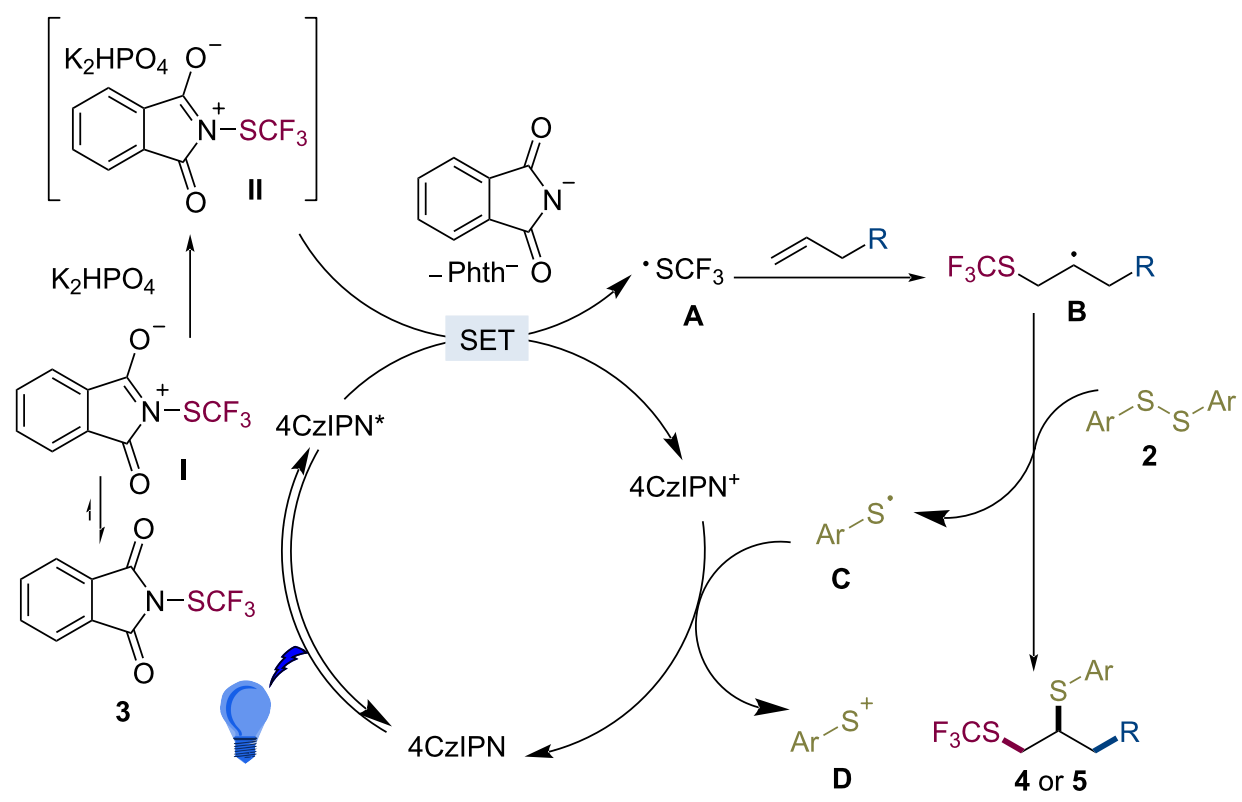




\section{References}

1. Yang, X.; Wu, T.; Phipps, R. J.; Toste, F. D. Chem. Rev. 2015, 115, 826-870. doi:10.1021/cr500277b

2. Wang, J.; Sánchez-Roselló, M.; Aceña, J. L.; del Pozo, C.; Sorochinsky, A. E.; Fustero, S.; Soloshonok, V. A.; Liu, H. Chem. Rev. 2014, 114, 2432-2506. doi:10.1021/cr4002879

3. O'Hagan, D. Chem. Soc. Rev. 2008, 37, 308-319. doi:10.1039/b711844a

4. Müller, K.; Faeh, C.; Diederich, F. Science 2007, 317, 1881-1886. doi:10.1126/science.1131943

5. Liang, T.; Neumann, C. N.; Ritter, T. Angew. Chem., Int. Ed. 2013, 52, 8214-8264. doi:10.1002/anie.201206566

6. Manteau, B.; Pazenok, S.; Vors, J.-P.; Leroux, F. R. J. Fluorine Chem. 2010, 131, 140-158. doi:10.1016/j.jfluchem.2009.09.009

7. Guo, S.; Cong, F.; Guo, R.; Wang, L.; Tang, P. Nat. Chem. 2017, 9 , 546-551. doi:10.1038/nchem.2711

8. Shao, X.; Hong, X.; Lu, L.; Shen, Q. Tetrahedron 2019, 75, 4156-4166. doi:10.1016/j.tet.2019.05.061

9. Hansch, C.; Leo, A.; Taft, R. W. Chem. Rev. 1991, 91, 165-195. doi:10.1021/cr00002a004

10. Hansch, C.; Leo, A.; Unger, S. H.; Kim, K. H.; Nikaitani, D.; Lien, E. J. J. Med. Chem. 1973, 16, 1207-1216. doi:10.1021/jm00269a003

11. Xu, C.; Ma, B.; Shen, Q. Angew. Chem., Int. Ed. 2014, 53, 9316-9320. doi:10.1002/anie.201403983

12. Glenadel, Q.; Alazet, S.; Billard, T. J. Fluorine Chem. 2015, 179, 89-95. doi:10.1016/j.jfluchem.2015.06.007

13. Ferry, A.; Billard, T.; Langlois, B. R.; Bacqué, E. Angew. Chem., Int. Ed. 2009, 48, 8551-8555. doi:10.1002/anie.200903387

14. Mukherjee, S.; Maji, B.; Tlahuext-Aca, A.; Glorius, F. J. Am. Chem. Soc. 2016, 138, 16200-16203. doi:10.1021/jacs.6b09970

15. Boiko, V. N. Beilstein J. Org. Chem. 2010, 6, 880-921. doi:10.3762/bjoc.6.88

16. Xu, X.-H.; Matsuzaki, K.; Shibata, N. Chem. Rev. 2015, 115, 731-764. doi:10.1021/cr500193b

17. Wu, H.; Xiao, Z.; Wu, J.; Guo, Y.; Xiao, J.-C.; Liu, C.; Chen, Q.-Y. Angew. Chem., Int. Ed. 2015, 54, 4070-4074. doi:10.1002/anie.201411953

18. Chen, Y.; Li, L.; Ma, Y.; Li, Z. J. Org. Chem. 2019, 84, 5328-5338. doi:10.1021/acs.joc.9b00339

19. Yin, G.; Mu, X.; Liu, G. Acc. Chem. Res. 2016, 49, 2413-2423. doi:10.1021/acs.accounts.6b00328

20. Wang, X.-X.; Lu, X.; He, S.-J.; Fu, Y. Chem. Sci. 2020, 11, 7950-7956. doi:10.1039/d0sc02054k

21. Tu, H.-Y.; Zhu, S.; Qing, F.-L.; Chu, L. Synthesis 2020, 52, 1346-1356. doi:10.1055/s-0039-1690842

22. Dhungana, R. K.; KC, S.; Basnet, P.; Giri, R. Chem. Rec. 2018, 18 , 1314-1340. doi:10.1002/tcr.201700098

23. Ji, M.; Wu, Z.; Yu, J.; Wan, X.; Zhu, C. Adv. Synth. Catal. 2017, 359, 1959-1962. doi:10.1002/adsc.201700218

24. Xiang, H.; Yang, C. Org. Lett. 2014, 16, 5686-5689. doi:10.1021/ol502751k

25. Zhu, L.; Wang, G.; Guo, Q.; Xu, Z.; Zhang, D.; Wang, R. Org. Lett. 2014, 16, 5390-5393. doi:10.1021/ol502624z

26. Xu, C.; Shen, Q. Org. Lett. 2015, 17, 4561-4563. doi:10.1021/acs.orglett.5b02315

27. Zhu, Z.; Luo, J.; Zhao, X. Org. Lett. 2017, 19, 4940-4943. doi:10.1021/acs.orglett.7b02406
28. Luo, J.; Liu, Y.; Zhao, X. Org. Lett. 2017, 19, 3434-3437. doi:10.1021/acs.orglett.7b01392

29. Xiao, Q.; He, Q.; Li, J.; Wang, J. Org. Lett. 2015, 17, 6090-6093. doi:10.1021/acs.orglett.5b03116

30. Luo, J.; Zhu, Z.; Liu, Y.; Zhao, X. Org. Lett. 2015, 17, 3620-3623. doi:10.1021/acs.orglett.5b01727

31. Jia, Y.; Qin, H.; Wang, N.; Jiang, Z.-X.; Yang, Z. J. Org. Chem. 2018, 83, 2808-2817. doi:10.1021/acs.joc.7b03261

32. Jiang, L.; Ding, T.; Yi, W.-b.; Zeng, X.; Zhang, W. Org. Lett. 2018, 20 , 2236-2240. doi:10.1021/acs.orglett.8b00581

33. Yang, T.; Lu, L.; Shen, Q. Chem. Commun. 2015, 51, 5479-5481. doi:10.1039/c4cc08655d

34. Fang, J.; Wang, Z.-K.; Wu, S.-W.; Shen, W.-G.; Ao, G.-Z.; Liu, F. Chem. Commun. 2017, 53, 7638-7641. doi:10.1039/c7cc01903c

35. Xiao, Z.; Liu, Y.; Zheng, L.; Zhou, X.; Xie, Y.; Liu, C.; Guo, Y.; Chen, Q.-Y. Tetrahedron 2018, 74, 6213-6219. doi:10.1016/j.tet.2018.09.019

36. Xiao, Z.; Liu, Y.; Zheng, L.; Liu, C.; Guo, Y.; Chen, Q.-Y. J. Org. Chem. 2018, 83, 5836-5843. doi:10.1021/acs.joc.8b00650

37. Yin, F.; Wang, X.-S. Org. Lett. 2014, 16, 1128-1131. doi:10.1021/ol403739w

38. Dagousset, G.; Simon, C.; Anselmi, E.; Tuccio, B.; Billard, T.; Magnier, E. Chem. - Eur. J. 2017, 23, 4282-4286. doi:10.1002/chem.201700734

39. Liu, Y.-L.; Qing, F.-L.; Xu, X.-H. Eur. J. Org. Chem. 2020, 1015-1018. doi:10.1002/ejoc.201901836

40. Liu, X.; Liang, Y.; Ji, J.; Luo, J.; Zhao, X. J. Am. Chem. Soc. 2018, 140, 4782-4786. doi:10.1021/jacs.8b01513

41. Saravanan, P.; Anbarasan, P. Chem. Commun. 2019, 55, 4639-4642. doi:10.1039/c9cc00815b

42. Li, H.; Shan, C.; Tung, C.-H.; Xu, Z. Chem. Sci. 2017, 8, 2610-2615. doi:10.1039/c6sc05093j

43. Ouyang, Y.; Xu, X.-H.; Qing, F.-L. Angew. Chem., Int. Ed. 2019, 58, 18508-18512. doi:10.1002/anie.201911323

44. Beletskaya, I. P.; Ananikov, V. P. Chem. Rev. 2011, 111, 1596-1636. doi:10.1021/cr100347k

45. Otsuka, S.; Nogi, K.; Yorimitsu, H. Top. Curr. Chem. 2018, 376, 13. doi:10.1007/s41061-018-0190-7

46. Li, H.; Liu, S.; Huang, Y.; Xu, X.-H.; Qing, F.-L. Chem. Commun. 2017, 53, 10136-10139. doi:10.1039/c7cc06232j

47. Honeker, R.; Garza-Sanchez, R. A.; Hopkinson, M. N.; Glorius, F. Chem. - Eur. J. 2016, 22, 4395-4399. doi:10.1002/chem.201600190

48. Xu, W.; Ma, J.; Yuan, X.-A.; Dai, J.; Xie, J.; Zhu, C. Angew. Chem., Int. Ed. 2018, 57, 10357-10361. doi:10.1002/anie.201805927

49. Pluta, R.; Nikolaienko, P.; Rueping, M. Angew. Chem., Int. Ed. 2014, 53, 1650-1653. doi:10.1002/anie.201307484

50. Rahman, M.; Buchspies, J.; Szostak, M. Catalysts 2019, 9, 129. doi:10.3390/catal9020129

51. Luo, J.; Zhang, J. ACS Catal. 2016, 6, 873-877. doi:10.1021/acscatal.5b02204

52. Jin, Y.; Yang, H.; Fu, H. Chem. Commun. 2016, 52, 12909-12912. doi:10.1039/c6cc06994k

53. Straathof, N. J. W.; Cramer, S. E.; Hessel, V.; Noël, T. Angew. Chem., Int. Ed. 2016, 55, 15549-15553. doi:10.1002/anie.201608297 


\section{License and Terms}

This is an Open Access article under the terms of the Creative Commons Attribution License (https://creativecommons.org/licenses/by/4.0). Please note that the reuse, redistribution and reproduction in particular requires that the author(s) and source are credited and that individual graphics may be subject to special legal provisions.

The license is subject to the Beilstein Journal of Organic Chemistry terms and conditions:

(https://www.beilstein-journals.org/bjoc/terms)

The definitive version of this article is the electronic one which can be found at:

https://doi.org/10.3762/bjoc. 17.49 